\title{
IMPENETRABLE SECURITY SYSTEM USING EMBEDDED SOFTWARES
}

\author{
BHASKAR. $\mathrm{S}^{1}$, SUMAN P. $\mathrm{M}^{2} \&$ SUDESH BHANDARI ${ }^{3}$ \\ ${ }^{1}$ Professor/ECE, S.J.C. Institute of Technology, Chickballapur, Karnataka, India \\ ${ }^{2,3}$ Student, BE, ECE, S.J.C. Institute of Technology, Chickballapur, Karnataka, India
}

\begin{abstract}
With the advent of digitalization, every consumer based services use computers and software as one of their basic requirement for efficient functioning. Now it is possible to bank at one's fingertips, shop with a swipe on a screen, travel with a click and much more. But with the steep rise in the use of digital technologies, it brings a new threat to security. Self-security also plays a vital role in this highly developing technological era, where authorization and ownership of lockers, banking and others must be secured. This project purposes a system that can effectively tackle security and theft issues using MATLAB and microcontroller with biometric scanner and GSM network technology.

KEYWORDS: Digitalization, Security, Authorization, GSM, Biometric Scanner \& Matlab
\end{abstract}

Received: May 20, 2019; Accepted: Jun 11, 2019; Published: Jun 27, 2019; Paper Id.: IJECIERDDEC20193

\section{INTRODUCTION}

The present age is a digital age. In today's world the term 'secure' and 'reliable' are of utmost importance. Typical security systems like password, voice recognition, thumbprint, palm scanning, bio- metric authentication may well be simply broken. A person is able to bypass the security of a system in many ways, knowing the system password or by use of violence or even by simply keeping the user in an unconscious state. Security is of utmost importance in these times. People are willing to pay huge amount of money to secure their most valuable items. The proposed project focuses on utilizing the current technologies to build a highly secure system that does not compromise with the security needs and worries of its user.

This proposed project, is a highly security system that gives the user the protection for their documents and offices/home with quality resolution and reliability. The security system is made up of three verification steps. The first step is the face identification of the user. Second is the fingerprint scan of the user. Once both the data are matched, a unique OTP is sent to the user's registered mobile number. The system unlocks once the OTP is entered to the system. This series of verification makes the system impenetrable and exclusive as well. Everybody has something precious to them and this project helps them safeguard it. The urgency for reliable security is of crucial concern. This circuit isn't designed for a security system in an exceedingly industrial application; however that ought to not forestall anyone from experimenting with it for that purpose.

\section{LITERATURE SURVEY}

Improving Biometric Authentication Performance from the User Quality Ajay Kumar, David Zhang. The effectiveness of a biometric measurement and sensing system is directly related to the performance generated from sensed data. This paper investigates a new approach to quantify the quality of sensed data from the user templates. The objective is to incorporate the quality of sensed data to generate a reliable estimate on the matching 
scores. The proposed method of extracting user quality is based on the confidence of generating reliable matching scores from the user templates. The experimental results of the proposed approach are also presented when the biometric measurements from the finger knuckles are employed. [1]

Security Issues in Biometric Authentication Qinghan Xiao User authentication is fundamental to the protection of information systems. The advantages of using biometrics to verify a person's identity over using passwords or tokens have been broadly presented. However, recent research has revealed that biometric technologies can be defeated with low-tech and cheap materials. Since biometrics are not secrets, there exists a risk of them being captured, copied, and forged. In this paper, the biometric authentication's vulnerability to various attacks is analyzed, and efforts to defeat the spoofing attacks are presented. In particular, multimodal biometric fusion approaches are presented to counteract security threats. [3]

Anil K. Jain In their paper they have explained about quality identification from face picture which uses two-dimensional face recognition approaches to acquire a decent performance only beneath strained environments. However, within the real applications, face look changes considerably because of totally different illumination, pose, and expression. Face recognizers supported representations of the input face pictures have different sensitivity to those variations. Therefore, a mixture of various face classifiers which may integrate the complementary info ought to cause improved classification accuracy. They use the sum rule and RBF-based integration methods to combine three normally used face classifiers based on PCA, ICA and LDA representations. [5]

Ethnicity Identification from Face Pictures Xiaoguang Lu and Anil

K. Jain In their paper they have explained about the human facial pictures that give the demographic info, like quality and gender. Conversely, quality and gender additionally play a crucial role in face-related applications. Image-based quality identification drawback is addressed during a machine learning framework. The Linear Discriminant Analysis (LDA) based theme is given for the two-class (Asian vs. non-Asian) quality classification task.[6]

Integrating Faces and Fingerprints for Personal Identification L. Hong and A.K. Jain An automatic personal identification system based solely on fingerprints or faces is often not able to meet the system performance requirements. The identity established by the system is more reliable than the identity established by a face recognition system. In addition, the proposed decision fusion scheme enables performance improvement by integrating multiple cues with different confidence measures. Experimental results demonstrate that our system performs very well.[11]

\section{OBJECTIVE OF THE PROJECT}

Our proposed project is to style, develop a system that may facilitate, build systems bases on face detection and recognition. This helps in finding major issues with respect to authentication whenever the user has got to be physically gifted. To be able to take live parameters of facial expression. To counteract the result of assorted parameters of traditional lighting condition encountered throughout recognition and authentication. Finger print identification exploitation using Biometric scanner. OTP generation victimization using GSM.

\section{Block Diagram and its Description}

As shown in Figure 1, the planned system consists of computer that provides the camera hardware as well as the software platform required to fulfill the proposed project. Matlab is used for the image processing required for the initial face recognition. Arduino microcontroller is used as the core hardware that combines the Biometric Scanner, GSM module 
and a DC motor. The microcontroller is responsible for the inter communication between the devices that will make possible of a system that is highly secure in real time.

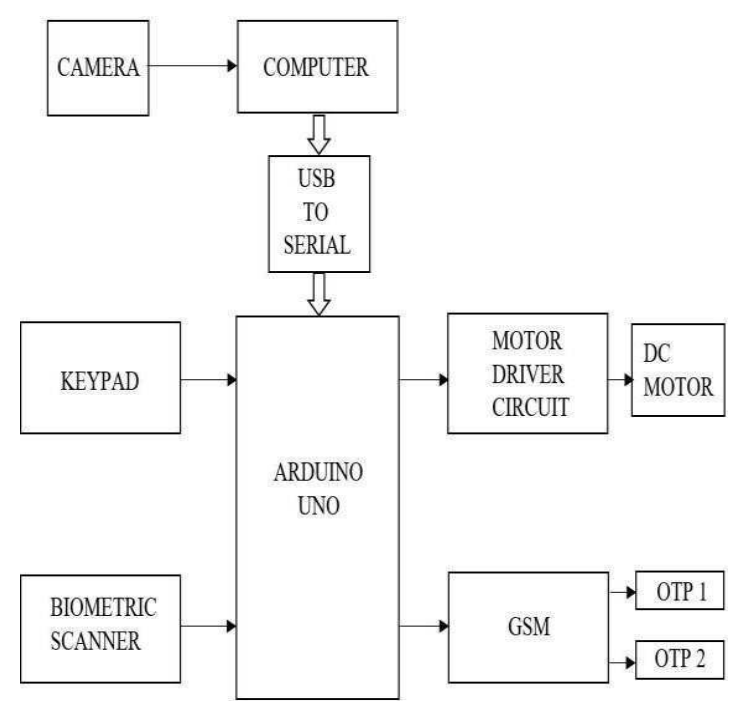

Figure 1: Block Diagram [2]

The system authenticates at the primary section with the face of the user which is recognized and detected. Once it detects the authenticated person, then it goes to second section where it scans the person's preferred finger impression and checks whether or not it matches. In third section it send OTP to the authenticated person's mobile through GSM technology, once the user enter the OTP then solely the lock can opened, which is showed by the rotation of the DC motor.

\section{METHODOLOGY}

Hardware requirements: Arduino Uno, Camera, Biometric scanner, Keypad, GSM, USB to serial, Motor driver circuit, DC motor.

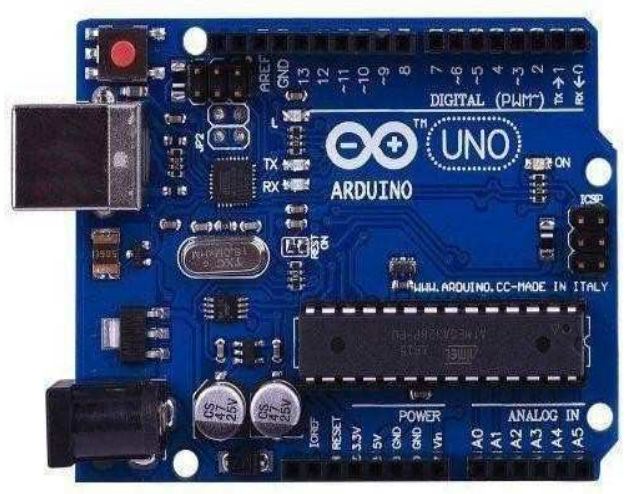

Figure 2: Arduino UNO [4]

Software Requirements: Matlab, Simulink, Arduino IDE 


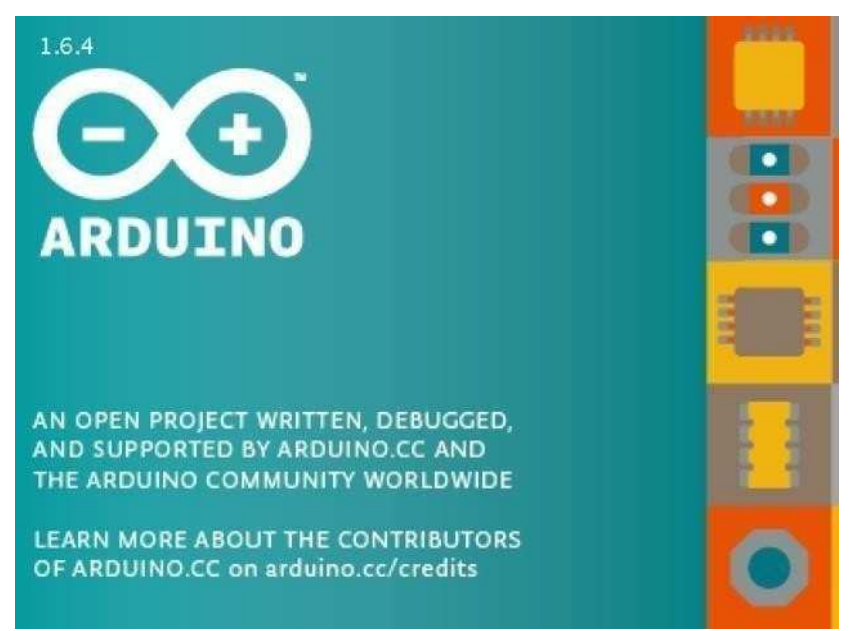

Figure 3: Arduino IDE [7]

\section{PROPOSED MODEL}

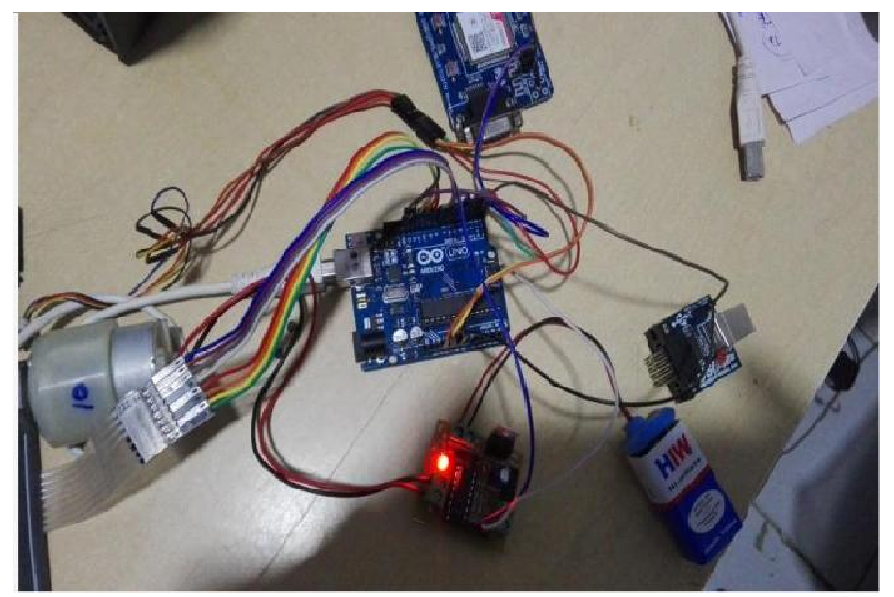

Figure 4: Proposed Model

In this model, Arduino microcontroller is interfaced with biometric scanner, GSM module and a motor driver circuit. The matlab code for face recognition is dumped to arduino board using serial to USB then which authorization is taken by biometric scanner, the code for biometric scanning is uploaded using arduino IDE and finally once the first two levels are crossed GSM module generates an OTP using some algorithms to the authorized person's mobile number. This model consists of a DC motor which is used as a system to be secured and unlocked with these three levels of security. The motor is driven by motor driver circuit which is also been interfaced with arduinouno microcontroller. If all the levels gets succeed then the motor rotates, if not the motor remains to be in its static position. 


\section{FLOWCHART}

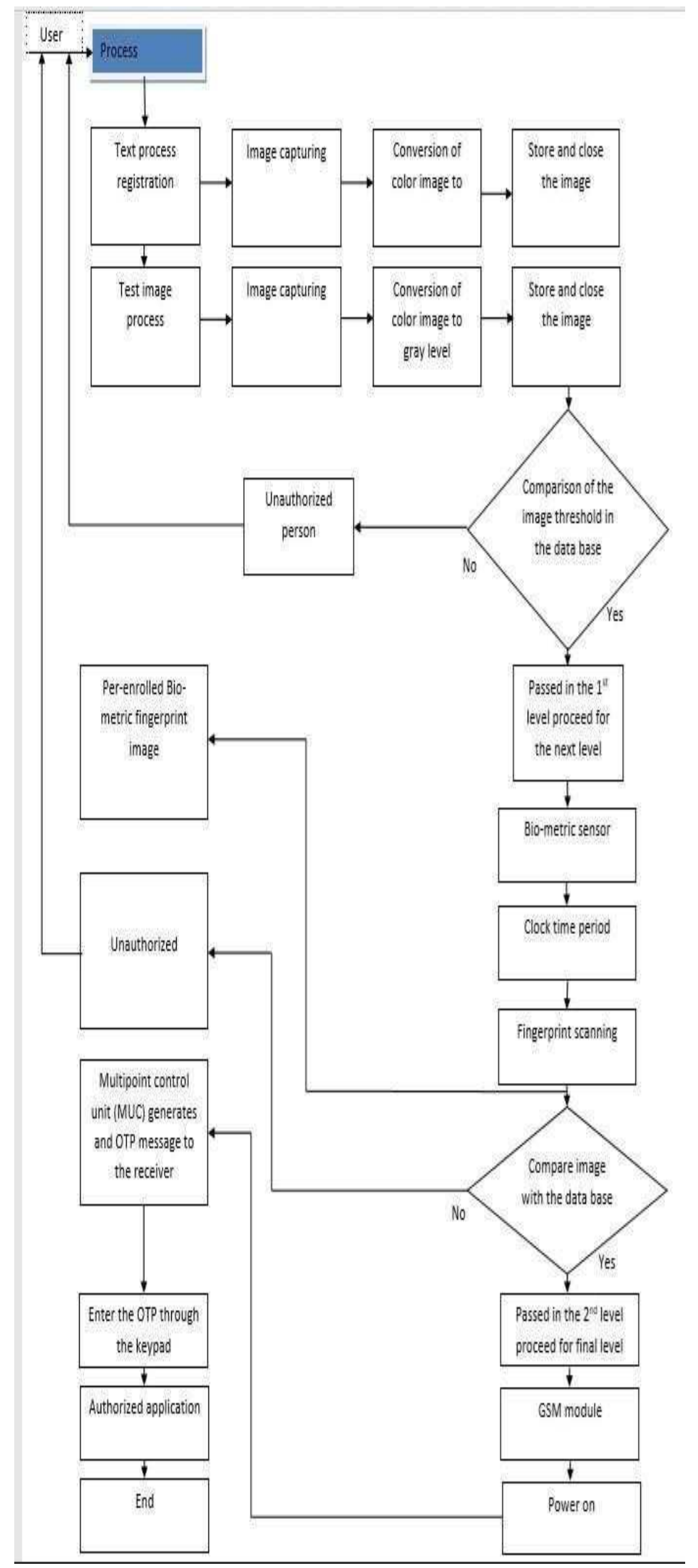

Figure 5: Flow Chart 


\section{APPLICATIONS AND ADVANTAGES}

\section{Applications}

Application of the project includes that it can enhance security system for military. Can be implemented as safe bank security locking system. Can be used for high protection ATM systems. Can be used in government offices. Can be used to safeguard national secrets and weapons.

\section{Advantages}

The advantages of the project is it provides high security, it can be used by an individual as well as multiple people. Also it is easy to setup. Provides no time fraud. Economically low cost. It is an upgradeable technology.

\section{CONCLUSIONS}

In the proposed model, the existing means of authentication devices were used in unison as to provide a near impenetrable security system. The project utilizes the knowledge of digital image processing to capture and verify an authenticated image. This project utilizes MATLAB for the image processing. Along with the image processing techniques, depth knowledge about the Arduino microcontroller and embedded programming enable interlink of the embedded systems that is the biometric and GSM along with the DC motor to make up this security system. The security system works on three levels of authentication. The first level is the face recognition of the user, the second level is the fingerprint authentication of the user. This level is enabled provided the first level is cleared.

Once the second level is cleared as well an OTP is sent to the authenticated mobile number making it the third and final level of the security authentication.

\section{REFERENCES}

1. Ajay Kumar, David Zhang, “Improving Biometric Authentication Performance From the User Quality”. IEEE Transactions on Instrumentation and Measurement, Volume 59, issue 3, March. 2010, pp 730 -735.

2. A.K. Jain, A. Ross and S. Pankanti, "Biometrics: A Tool for Information Security", IEEE Transactions on Information Forensics And Security, Volume1, issue 2, Jun. 2006, pp 125 -144.

3. A. Ross, S. Dass, and A. K. Jain, “A deformable model for fingerprint matching”, Journal of Pattern Recognition, Elsevier, Volume 38, No. 1, Jan. 2005, pp.95-103.

4. J. L. Wayman, A. K. Jain, D. Maltoni, and D. Maio, Eds.,

5. “Biometric Systems: Technology, Design and Performance Evaluation”, New York: Springer Verlag, 2005.

6. Xiaoguang. Lu, Rein Lien Hsu, and A.K. Jain. "Face Recognition with 3D Model- Based Synthesis”. Proc. International Conference on Biometric Authentication, LNCS 3072. Hong Kong, July2004.

7. X. Lu, D. Colbry, and A.K. Jain. “Three-Dimensional Model Based Face Recognition”. Proc. International Conference on Pattern Recognition. Cambridge, UK, August2004.

8. A. K. Jain, A. Ross, and S. Prabhakar, "An introduction to biometric recognition," IEEE Trans. Circuits Syst. Video Technology, Special Issue Image- and VideoBased Biomet., Volume 14, Issue 1, Jan. 2004, pp.4-20.

9. Anil K. Jain, “Face Detection and Recognition”, OMB No. 0704- 0188,2004.

10. Xiaoguang Lu, Anil K. Jain, “Ethnicity identification from face images”, Published 2004. 
11. X. Lu, D. Colbry, and A.K. Jain. "Matching 2.5D Scans for Face Recognition”. Proc. International Conference on Biometric Authentication, LNCS 3072. Hong Kong, July2004.

12. Priyadharshini, A. (2013). A survey on security issues and countermeasures in cloud computing storage and a tour towards multi-clouds. International Journal of Research in Engineering \& Technology, 1(2), 1-10.

13. X. Lu, R.L. Hsu, and A.K. Jain. "Resampling for Face Recognition”. Proc. International Conference on Audio- and VideoBased Biometric Person Authentication (A VBPA '03). Guildford, UK June 2003. Audio- and Video-Based Biometric Person Authentication (AVBPA 2003). Guildford.

14. Y. Wang, X. Lu and A.K. Jain, "Combining classifiers for face recognition", 2003 International Conference on Multimedia and Expo. ICM '03. Proceedings (Cat. No.03TH8698)(ICME), Baltimore, MD, USA, 2003,pp.13-1 
